\section{Fludrocortisone for Heparin-Induced Hyperkalemia}

Heparin, which continues to be used widely for prophylaxis and treatment of thromboembolism, is associated with a risk of hyperkalemia. ${ }^{1}$ Clinicians should be aware of strategies to address heparin-induced hyperkalemia. The following account supports the utility of fludrocortisone in normalizing renal processing of potassium during heparin therapy.

A patient* was transferred from a peripheral community hospital for syncope associated with complete heart block (heart rate $39-45 / \mathrm{min})$. On the evening of admission, intubation and mechanical ventilation were required for hypercapnic respiratory failure. A permanent pacemaker was inserted the next morning, which increased the patient's heart rate and improved signs of perfusion to the vital organs (kidney, brain, and skin). Elevated serum creatinine at the time of admission was thought to be a sign of impaired renal perfusion secondary to the heart block. Given the patient's body habitus, weaning from mechanical ventilation was prolonged, but extubation to biphasic positive airway pressure (BiPAP) via a face mask was successful on day 12 of the admission. During the weaning process, renal function improved, as indicated by declining concentrations of serum creatinine (Figure 1).

*The patient's consent for publication was not obtained. All identifying information has been removed from this letter.
The patient's large body size led to initiation, upon admission, of a prophylactic regimen for deep vein thrombosis, consisting of heparin 30000 units per $24 \mathrm{~h}$, given as 10000 units SC q8h, based on the following equation: [0.66 $\times(71.34$ $\times$ weight in kilograms $)+(83.75 \times$ height in inches $)-$ 3467.59)].2

Despite an improvement in renal function, the concentration of serum potassium rose during the hospital stay (Figure 1). No exogenous potassium supplementation was administered, beyond the potassium included in the enteral nutrition solution. Sodium polystyrene $30 \mathrm{~g}$ was administered rectally on day 13 and orally on days 14 and 15 . The transtubular potassium gradient (TTKG) was calculated to assess the factors contributing to the hyperkalemia; this analysis suggested a state of relative hypoaldosteronism. ${ }^{3}$ More specifically, the TTKG was 2.5, based on serum potassium $6 \mathrm{mmol} / \mathrm{L}$, urine potassium $30 \mathrm{mmol} / \mathrm{L}$, serum osmolality $319 \mathrm{mmol} / \mathrm{L}$, and urine osmolality $634 \mathrm{mmol} / \mathrm{L}$. Serum aldosterone concentration measured on day 13 was $96 \mathrm{pmol} / \mathrm{L}$ (normal range for patient in supine position $30-415 \mathrm{pmol} / \mathrm{L}$ ), which supported the diagnosis of a state of relative hypoaldosteronism. The diagnosis was further supported by the calculation of plasma aldosterone/(plasma potassium - 4.2), which was less than 280 (patient data: 96/[5.5 - 4.2]). ${ }^{4}$ The serum renin concentration was only slightly elevated $(0.35 \mathrm{ng} / \mathrm{L} / \mathrm{s}$; normal value for patient in the supine position $<0.28 \mathrm{ng} / \mathrm{L} / \mathrm{s}$ ), and the serum cortisol level was normal (436 nmol/L; normal range $175-685 \mathrm{nmol} / \mathrm{L}$ ). To overcome the

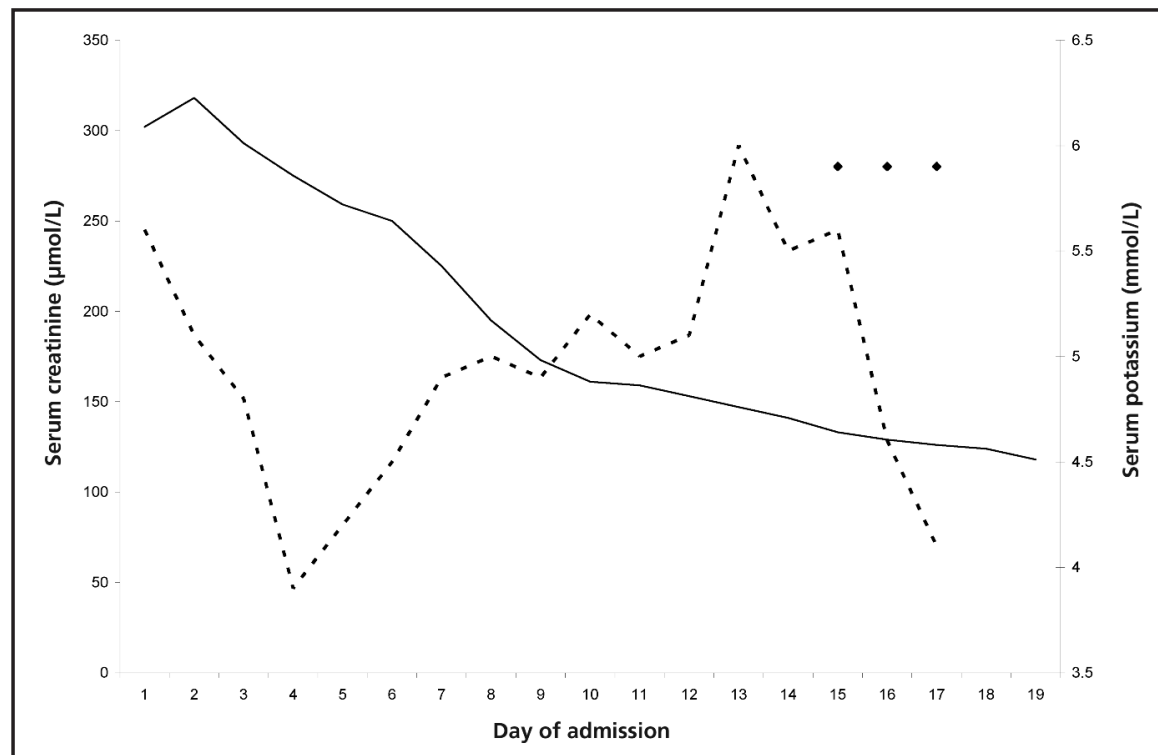

Figure 1. Serum levels of creatinine (solid line) and potassium (dashed line) over the course of the patient's stay in hospital. Three doses of fludrocortisone were administered (solid diamonds). 
heparin-induced hypoaldosterone state, fludrocortisone $0.1 \mathrm{mg}$ once daily was given orally on days 15,16 , and 17 . The serum potassium decreased rapidly with the fludrocortisone (Figure 1). The patient was discharged to the peripheral community hospital on day 19 for further rehabilitation.

It has been known for over 50 years that heparin can impair the excretion of potassium. ${ }^{5}$ The mechanism of this inhibition is thought to be suppression of aldosterone synthesis through a reduction in the number and affinity of angiotensin II receptors in the adrenal zona glomerulosa. ${ }^{5}$ This process dampens the stimulation by the renin-angiotensin system of retention of sodium and excretion of potassium. Because corticosteroids can stimulate sodium retention and potassium excretion by interacting with receptors independent of angiotensin II, exogenous mineralocorticoids remain effective. ${ }^{5}$ This mechanism does not alter the production of renin or corticotropin, consistent with the findings of the case reported here (in which serum renin and cortisol concentrations remained normal). The maximum antagonism of aldosterone effects by heparin occurs after 4-6 days of therapy and may occur at any dosage in clinical use. ${ }^{5}$ In this case, the Naranjo scale was used to assess the probability of an adverse drug reaction. The Naranjo score of 6 suggested that the hyperkalemia was probably due to the heparin.

Hyperkalemia caused by heparin therapy is reported to occur in about $7 \%$ of patients. ${ }^{5}$ Substitution with lowmolecular-weight heparins (LMWHs) is not an effective strategy to prevent hyperkalemia, because the LMWHs are also associated with a clinically significant incidence of hyperkalemia. ${ }^{6}$ The effect of nonheparin anticoagulants on potassium control in morbidly obese, critically ill patients is unknown, as is the appropriate dosage of alternative therapies, such as the activated factor $\mathrm{Xa}$ inhibitor fondaparinux. ${ }^{7}$ Alternatively, the hypoaldosterone state induced by heparin may be antagonized by fludrocortisone. ${ }^{5,8,9}$ Clinicians should consider the short-term use of fludrocortisone in hyperkalemic patients until the need for heparin therapy elapses.

\section{References}

1. Mitchell S, Bayliff CD, McCormack DG. Heparin induced hyperkalemia. Can J Hosp Pharm 1993;46(3):125-127.

2. Shepherd MF, Rosborough TK, Schwartz ML. Heparin thromboprophylaxis in gastric bypass surgery. Obes Surg 2003;13(2):249-253.

3. Choi MJ, Ziyadeh FN. The utility of the transtubular potassium gradient in the evaluation of hyperkalemia. J Am Soc Nephrol 2008;19(3):424-426.

4. Adam WR. Hypothesis: A simple algorithm to distinguish between hypoaldosteronism and renal aldosterone resistance in patients with persistent hyperkalemia. Nephrology (Carlton) 2008;13(6):459-464.

5. Oster JR, Singer I, Fishman LM. Heparin-induced aldosterone suppression and hyperkalemia. Am J Med 1995;98(6):575-586.

6. Gheno G, Cinetto L, Savarino C, Vellar S, Carraro M, Randon M. Variations of serum potassium level and risk of hyperkalemia in inpatients receiving lowmolecular-weight heparin. Eur J Clin Pharmacol 2003;59(5-6):373-377.

7. Cumbo-Nacheli G, Samavati L, Guzman JA. Bioavailabilitly of fondaparinux to critically ill patients. J Crit Care 2011;26(4):342-346.

8. DeFronzo RA. Hyperkalemia and hyporeninemic hypoaldosteronism. Kidney Int 1980;17(1):118-134.

9. Sherman DS, Kass CL, Fish DN. Fludrocortisone for the treatment of heparin-induced hyperkalemia. Ann Pharmacother 2000;34(5):606-610.

Glen Brown, PharmD, FCSHP, BCPS(AQ)

Pharmacy Department

St Paul's Hospital

Vancouver, British Columbia

\begin{tabular}{|c|c|c|}
\hline & Ad Page & Prescribing Information \\
\hline CPhA / ADAPT Online Course & 398 & - \\
\hline Hospira / Docetaxel & 394 & - \\
\hline Hospira / Precedex & 392,393 & 465,466 \\
\hline Medbuy / Corporate & IBC & - \\
\hline Pharmaceutical Partners of Canada / Corporate & IFC & - \\
\hline Pharmaceutical Partners of Canada / Corporate & $\mathrm{OBC}$ & - \\
\hline Sandoz / Corporate & 386 & - \\
\hline Sandoz / Corporate & 388 & - \\
\hline
\end{tabular}

